\title{
SOME ESTIMATES OF HARDY OPERATORS AND THEIR COMMUTATORS ON MORREY-HERZ SPACES
}

\author{
GUILIAN GAO AND YONG ZHONG
}

\begin{abstract}
In this paper, we study the boundedness of $p$-adic Hardy operators $\mathscr{H}^{p}$ and $\mathscr{H}^{p, *}$ on the $p$-adic Morrey-Herz space. Furthermore, we establish the Lipschitz estimates for commutators of $p$-adic Hardy operators $\mathscr{H}_{b}^{p}$ and $\mathscr{H}_{b}^{p \text {,* }}$ on the $p$-adic function spaces, such as the $p$-adic Lebesgue space, the $p$-adic Herz space and the $p$-adic Morrey-Herz space. Moreover, we also obtain the $C M O$ estimates. All our results are also true for the fractional $p$-adic Hardy operator.
\end{abstract}

Mathematics subject classification (2010): 42B35, 47G10.

Keywords and phrases: $p$-adic Hardy operator, $p$-adic Morrey-Herz space, Jensen's inequality.

\section{REFERENCES}

[1] M. Christ, L. Grafakos, Best constants for two nonconvolution inequalities, Proc. Amer. Math. Soc., 123 (1995), 1687-1693.

[2] N. M. Chuong, D. V. Duong, Weighted Hardy-Littlewood operators and commutators on p-adic functional spaces, $p$-Adic Numb. Ultr. Anal. Appl., 5 (2013), 65-82.

[3] N. M. Chuong, Yu. V. Egorov, A. Yu. Khrennikov, Y. Meyer, D. Mumford, Harmonic, Wavelet and p-Adic Analysis, World Scientific, (2007).

[4] N. M. Chuong, H. D. Hung, Maximal functions and weighted norm inequalities on local fields, Appl. Comput. Harmon. Anal., 29 (2010), 272-286.

[5] D. E. Edmunds, W. D. Evans, Hardy Operators, Function Spaces and Embeddings, Springer Verlag, Berlin, (2004).

[6] W. G. FARIS, Weak Lebesgue spaces and quantum mechanical binding, Duke Math. J., 43 (1976), 365-373.

[7] Z. W. Fu, L. Grafakos, S. Z. Lu AND F. Y. Zhao, Sharp bounds for $m$-linear Hardy and Hilbert operators, Houston J. Math., 38 (2012), 225-244.

[8] Z. W. FU, Z. G. LiU, S. Z. LU AND H. B. WANG, Characterization for commutators of ndimensiaonal fractional Hardy operators, Sci. China Ser. A Math. 50 (2007), 1418-1426.

[9] Z. W. FU, Q. Y. WU AND S. Z. LU, Sharp estimates of p-adic Hardy and Hardy-Littlewood-Pólya operators, Acta Math. Sinica, 29 (2013), 137-150.

[10] G. H. HARDY, Note on a theorem of Hibert, Math. Z., 6 (1920), 314-317.

[11] G. H. Hardy, J. E. LitTlewood and G. Pólya, Inequalities, second edtion, Cambridge Univ. Press, London, (1952).

[12] Y. C. RIM, Carleson measures and the BMO space on the p-adic vector space, Math. Z., 282 (2009), 1470-1477.

[13] K. S. RIM AND J. LEE, Estimates of weighted Hardy-Littlewood averages on the p-adic vector space, J. Math. Anal. Appl., 324 (2006), 1278-1304.

[14] M. Taibleson, Fourier Analysis on Local Fields, Princeton Univ. Press, Princeton, (1975).

[15] V. S. Vladimirov and I. V. Volovich, p-Adic quantum mechanics, Comm. Math. Phys., 123 (1989), 659-676.

[16] V. S. Vladimirov, I. V. Volovich And E. I. Zelenov, p-Adic Analysis and Mathematical Physics, World Scientific, Singapore, 1994. 
[17] S. S. Volosivets, Hausdorff operators of special kind in Morrey and Herz p-adic spaces, p-adic numbers, Ultrametric Anal. Appl., 4 (2012), 222-230.

[18] S. M. WANG, D. Y. YAN, Weighted boundedness of commutators of fractional Hardy operators with Besov-Lipschitz functions, Anal. Theory Appl., 28 (2012), 79-86.

[19] Q. Y. WU, Z. W. FU, Sharp estimates of m-linear p-adic Hardy and Hardy-Littlewood-Pólya operators, J. Appl. Math., 2011 (2011), Art. ID 472176, 20 pp.

[20] Q. Y. WU, Z. W. FU, Hardy-Littlewood-Sobolev inequalities on p-adic central Morrey spaces, J. Funct. Spaces, 2015 (2015), Art. ID 419532, 7 pp.

[21] Q. Y. WU, Z. W. FU, Weighted p-adic Hardy operators and their commutators on p-adic central Morrey spaces, Bull. Malays. Math. Sci. Soc., in press.

[22] Q. Y. WU, L. MI AND Z. W. FU, Boundedness of p-adic Hardy operators and their commutators on p-adic central Morrey and BMO spaces, J. Funct. Spaces Appl., 2013 (2013), Art. ID 359193, 10 pp.

[23] Q. Y. WU, Boundedness for commutators of fractional p-adic Hardy operators, J. Ineq. Appl., 2012 (2012): 293. 